\title{
Características de frutos de café sob compressão ${ }^{1}$
}

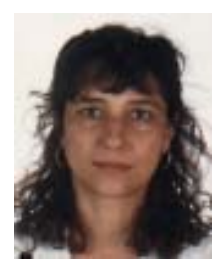

Sandra M. Couto ${ }^{2}$, Cláudia da S. Batista ${ }^{3}$, Ivano A. Devilla ${ }^{4} \&$ Vivian T. Paim ${ }^{5}$

1 Trabalho financiado pelo Consórcio Brasileiro de Pesquisa e Desenvolvimento em Café - CBP\&D Café

2 DEA/UFV. Av. Ph Rolfs, s/n. CEP 36571-000, Viçosa, MG. Fone: (31) 3899-1924. E-mail: scouto@mail.ufv.br (foto)

${ }^{3}$ DEA/UFV. Fone: (31) 3891-9149. E-mail: cbatista@buynet.com.br

4 DEA/UFV. Fone: (31) 3891-2927. E-mail: devilla@buynet.com.br

5 DEA/UFV. Fone: (31) 9965-0353

Protocolo $146-21 / 2 / 2000$

\begin{abstract}
Resumo: As condições necessárias para a realização de ensaios de compressão em frutos de café visando à obtenção de resultados confiáveis das forças envolvidas no colapso do produto, foram investigadas neste trabalho e determinados os requerimentos de forças, deformações e energias para o colapso do material sob compressão. As variáveis independentes investigadas foram: presença do pedúnculo no fruto, velocidade de compressão, grau de maturação dos frutos e posição do produto durante a compressão. Os frutos testados encontravam-se em três estádios de maturação: 'cereja', 'verdoengo' e 'verde'. As análises dos resultados mostraram que, para se ter um erro menor ou igual a 10\% na estimativa das forças de colapso do produto, são necessárias amostras de, pelo menos, cinqüenta frutos. Os valores das forças, deformações específicas e energias, para o colapso de frutos 'cereja', não foram afetados pela presença do pedúnculo no produto. A velocidade de compressão parece não influenciar nos valores das grandezas investigadas; já a posição do produto, ao ser comprimido, e o estádio de maturação do fruto, mostraram ser variáveis significativas.
\end{abstract}

Palavras-chave: Coffea arabica L., forças, deformação, colapso

\section{Characteristics of coffee fruits under compression load}

\begin{abstract}
Basic conditions required to obtain accuracy on the values of forces for collapse of coffee fruits under compression tests were investigated. The requirements of force, strain and energy up to the collapse point of the material were also determined. The independent variables investigated were: presence of peduncle; compression velocity; product maturation stage and fruit orientation during the compression. The fruits tested were at three maturation stages (classified by color): 'cherry', 'green' and one named 'verdoengo' (stage somewhere between cherry and green). Analysis of the results showed a sample size requirement of, at least, fifty fruits to estimate the collapse forces with an error less than $10 \%$. The values of forces, strains and energies up to the collapse of 'cherry' fruits were not influenced by the presence of peduncle on their surfaces. Also the compression velocity seems to have no influence on the collapse parameters investigated. On the other hand, fruit orientation and product maturation stage were found to affect the values of force, strain and energy up to the fruit collapse.
\end{abstract}

Key words: Coffea arabica L., force, strain, collapse

\section{INTRODUÇÃO}

Da produção ao consumo, produtos agrícolas ficam sujeitos à ação de forças estáticas e dinâmicas durante as várias operações e processamentos aos quais são submetidos. Os processos físicos e químicos requeridos para conversão de um material em um produto envolvem, geralmente, várias operações mecânicas; assim, na investigação do comportamento mecânico de um produto é essencial identificar-se e quantificar as propriedades pertinentes ao processo ao qual este será submetido.

Uma plantação de milho, por exemplo, pode ter finalidades diferentes, como silagem para alimentação de animais, grãos para alimentação ou para processamento (farinha, óleo, margarina etc). Se colhido verde como silagem, as propriedades mecânicas pertinentes são: resistência do caule ao corte, propriedades aerodinâmicas para o seu escoamento e transporte, características de compactação etc, mas, se colhido como grão, 
além de algumas já citadas, serão necessárias outras, como resistência à separação do grão da espiga (debulha), aos danos mecânicos, à moagem, à extrusão e às operações de classificação.

Portanto, para o manuseio adequado de um produto, torna-se essencial o conhecimento prévio das características físicas e propriedades mecânicas que tenham relevância e que possam ser aplicadas diretamente no desenvolvimento de máquinas destinadas à colheita, ao transporte, ao beneficiamento e ao armazenamento.

O café é um produto agrícola altamente caracterizado pela sua qualidade final, que depende dos diversos processos empregados na colheita e na pós-colheita. Dentre os parâmetros mais importantes que influenciam a qualidade do café, citam-se características da espécie e variedade, condições ambientais durante o seu desenvolvimento, época e procedimento de colheita, método de secagem e práticas de armazenagem (Silva \& Berbert., 1999). Segundo Amorim et al. (1977) a qualidade do café se deteriora, tanto física como em termos das características organolépticas, durante a colheita, processamento e armazenamento (as paredes celulares do produto tornam-se mais finas, a densidade diminui, a cor passa de verde para branco, amarelo ou marrom e a atividade de algumas enzimas decresce e aumentam os teores de proteína de baixo peso molecular).

A colheita é a operação de maior custo para o cafeicultor, difícil de ser realizada e, quando feita manualmente, demanda uma quantidade excessiva de mão-de-obra. A qualidade do café está diretamente associada ao processo de colheita a qual, quando mecânica, pode tornar-se importante fator para a obtenção de um produto de melhor qualidade e para a minimização dos problemas da escassez de mão-de-obra no período da colheita.

Poucas são as informações sobre as propriedades mecânicas do café e os parâmetros que afetam suas propriedades e resistência a danos mecânicos. Pesquisas com outros tipos de grãos agrícolas mostram que o comportamento mecânico desses produtos é afetado principalmente pelos seguintes parâmetros: variedade, teor de umidade, tipo de força ao qual o produto é submetido e região no grão de aplicação dessas forças (Mohsenin, 1978; Prussia \& Campbell, 1985; Li et al., 1989; Zhang et al., 1989; Gupta \& Das, 2000; Henry et al., 2000); é clássico, também, o conhecimento de que, quando submetidos a forças de intensidades moderadas, os grãos com baixos teores de umidade exibem características de sólidos elásticos, enquanto aqueles com altos teores de umidade se comportam como materiais viscoelásticos, ou seja, suas propriedades são dependentes do tempo ou da taxa de aplicação de forças.

O comportamento mecânico individual de frutos e grãos de café é, provavelmente, dependente, dentre outros, de parâmetros similares àqueles pertinentes aos outros tipos de grãos; entretanto, existem certos aspectos que tornam o café diferente. Durante o processo de colheita mecanizada, por exemplo, a variação do teor de umidade dos grãos (mais comuns) em uma área é pequena, o que não acontece com o café. No Brasil, por razões climáticas, não há controle sobre o estádio de maturação do fruto durante a colheita e, por razões econômicas, colhem-se frutos que variam, em toda a sua faixa de classificação, desde o "verde" até o "coco". A determinação das forças que os mecanismos de colheita precisam fornecer para que os frutos, em diferentes estádios de maturação, se desprendam da planta, das cargas que o material poderá suportar sem sofrer danos e das resistências relativas dos frutos de café, faz-se necessária para o desenvolvimento de máquinas e metodologias visando à minimização da perda de qualidade do café.

$\mathrm{Na}$ colheita de café, conforme o estádio de maturação dos frutos, pode ocorrer a perda de integridade da casca do produto; estes, por sua vez, podem ser destacados, com ou sem o pedúnculo das plantas. Observa-se que a perda do pedúnculo, durante a colheita, quando o fruto se encontra a um teor de umidade mais alto (principalmente na coloração “cereja”) resulta em danos na sua casca, o que provoca o vazamento de líquidos do seu interior. Esses danos no envoltório podem contribuir para mudanças nos valores dos parâmetros envolvidos na ruptura do produto.

Com base no exposto e como uma primeira aproximação, o comportamento mecânico de frutos de café poderia ser considerado dependente dos seguintes parâmetros: variedade do produto, estádio de maturação dos frutos na colheita, tipo de força que atuará sobre o material, velocidade de aplicação das forças e local de aplicação dessas forças no fruto. Desta forma, este trabalho teve, por objetivo, investigar as condições básicas necessárias à realização de ensaios de compressão em frutos de café, visando à obtenção de resultados confiáveis das forças envolvidas no colapso do produto e determinar os requerimentos de forças, deformações e energias para o colapso do material submetido a compressões. Foram investigadas as influências, nesses requerimentos, das seguintes variáveis: (a) presença do pedúnculo no fruto; (b) velocidade de compressão; (c) grau de maturação dos frutos e (d) posição do produto durante a compressão.

\section{MATERIAL E MÉTODOS}

$\mathrm{Na}$ determinação dos valores das forças, deformações e energias envolvidas no colapso, por compressão, de frutos de café, variedade Catuaí Vermelho, o produto foi colhido manualmente de plantas existentes na área de Armazenamento do Departamento de Engenharia Agrícola/UFV. Procurou-se colher frutos nas cores mais pronunciantes de "cereja", "verdoengo" e "verde" (detecção visual). Testes de compressão uniaxiais nos frutos foram realizados em uma máquina de ensaios universal (EMIC) utilizando-se uma célula de carga de $980 \mathrm{~N}$.

Das curvas de compressão do produto (força versus deformação, provenientes da máquina de ensaios) foram obtidas as seguintes informações: valores da força máxima sustentada pelo fruto antes do colapso; da deformação específica do produto ao atingir esta força, e da energia requerida para o colapso do produto. As forças máximas foram obtidas diretamente das curvas e a deformação específica foi determinada pela relação entre a deformação do fruto após compressão (deslocamento da placa compressora da máquina) e o seu comprimento inicial, todos medidos segundo o eixo de compressão. A energia para o colapso do fruto foi obtida através da determinação da área sob a curva de força versus deformação, até o ponto de interesse.

Geralmente, a finalização de um ensaio de compressão pode ocorrer de duas maneiras: (a) por ruptura ou (b) por colapso; 
no primeiro caso, a força atuante em um corpo, após atingir determinado valor, sofre queda brusca até chegam a valor igual a zero, enquanto no segundo caso a força atuante no material atinge um valor máximo e, a seguir, cai lentamente, até atingir valor nulo ou tem sua intensidade reduzida, mas não alcança um valor nulo. A última situação é a que ocorre com frutos de café; as forças atuantes durante a compressão do produto atingem valor máximo e, depois, têm a intensidade reduzida, indicando a ocorrência de algum colapso na estrutura do fruto. Nos ensaios realizados cada teste foi encerrado quando a força atuante no fruto atingiu valor $5 \%$ menor que a intensidade da força máxima percebida pelo material.

Na determinação de parâmetros relacionados com a resistência de um produto a cargas compressivas, a velocidade de aplicação das forças deve basear-se na sensibilidade do material à taxa de carregamento e se especificam, como padrão, as seguintes faixas de velocidade: (a) de 2,5 a $30 \mathrm{~mm} \mathrm{~min}^{-1}$, para frutos e vegetais duros; (b) abaixo de $10 \mathrm{~mm} \mathrm{~min}^{-1}$, para frutos e vegetais macios e (c) de $1,25 \mathrm{~mm} \mathrm{~min}^{-1} \pm 50 \%$, para sementes e grãos (Agricultural Engineers Yearbook of Standards, 1995). Neste trabalho foram usadas as seguintes velocidades de compressão: 0,5, 2,5, 5,0, 7,5 e $10 \mathrm{~mm} \mathrm{~min}^{-1}$.

$\mathrm{Na}$ investigação do grau de dependência entre as intensidades das forças necessárias à ruptura do produto e o seu posicionamento, considerou-se a existência de dois planos ortogonais de simetria (xy e xz) no fruto, como esquematizados na Figura 1. A resistência mecânica do produto foi determinada para forças aplicadas segundo as direções P1 (posição mais estável do fruto) P2 e P3.

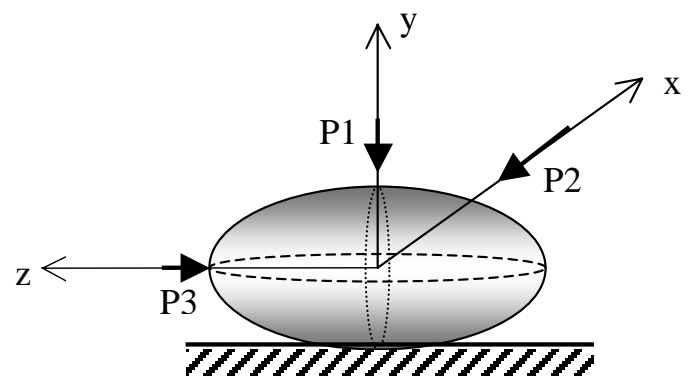

Figura 1. Prováveis planos de simetria do fruto de café e posições de compressão

Dois tipos de experimento, denominados no texto "Experimento 1" e "Experimento 2", foram realizados e as metodologias adotadas são descritas nos itens que seguem.

\section{Experimento 1}

Neste experimento, trabalhou-se com frutos de café nos estádios de maturação "cereja" e "verde", investigaram-se os efeitos de presença de pedúnculo em frutos "cereja", velocidade de compressão do produto nos valores das forças, deformações e energias para o colapso do material e, também, a célula de carga mais adequada para ensaios de compressão de café (para maior exatidão nos resultados) e o tamanho da amostra a ser comprimida, para assegurar, com 95\% de confiança, que o erro na estimativa da força para o colapso dos frutos fosse menor ou igual a $10 \%$.

Para execução dos testes de compressão, os frutos cor "cereja" foram retirados da planta com o pedúnculo e sem o pedúnculo, enquanto com os frutos cor "verde", não houve esta preocupação.

Em todos os testes foram realizados ensaios com 20 frutos e cada fruto foi comprimido, segundo sua posição mais estável (P1, Figura 1), pelas placas rígidas e paralelas da máquina de ensaios. As velocidades de compressão usadas foram de 0,5 , 2,5, 5,0, 7,5 e $10 \mathrm{~mm} \mathrm{~min}^{-1}$. Os ensaios foram realizados imediatamente após a colheita e os teores de umidade dos frutos "verde" e "cereja" encontravam-se a $(75,3 \pm 0,1)$ e $(65 \pm 2) \%$ b.u., respectivamente.

\section{Experimento 2}

Aqui, as variações nos valores das forças, deformações e energias para colapso do material, foram determinadas em função do grau de maturação dos frutos e local de aplicação, no produto, das forças de compressão. Os frutos usados neste experimento encontravam-se em três estádios de maturação: cereja, a $69,9 \pm 0,4 \%$ b.u.; verdoengo, a $75,7 \pm 0,9 \%$ b.u., e verde, a $72,0 \pm 0,8 \%$ b.u. Em cada teste foram comprimidos 50 frutos a uma velocidade de $5 \mathrm{~mm} \mathrm{~min}^{-1}$. Três posições do produto durante a compressão, esquematizadas na Figura 1, foram testadas. Neste experimento não houve a preocupação de seleção dos frutos quanto à presença de pedúnculo.

\section{RESULTADOS E DISCUSSÃO}

\section{Experimento 1}

Presença de pedúnculo em frutos “cereja”: a Figura 2 apresenta os valores médios de forças, deformações específicas e energias requeridas para o colapso dos frutos de café "cereja", colhidos com e sem pedúnculo, após a compressão a diferentes velocidades. Para frutos com pedúnculo, as forças médias variaram de 34,3 a 61,7 N, com desvios na faixa de 14 a $42 \mathrm{~N}$, enquanto para frutos sem pedúnculo as forças médias variaram de 30,0 a 58,6 N, com desvios variando de 12 a 25 N. A energia média requerida para o colapso de frutos com pedúnculo variou de 41,4 a 75,7 $\mathrm{N} \mathrm{mm}$, com desvios na faixa de 23 a $75 \mathrm{~N} \mathrm{~mm}$, enquanto aquela requerida para frutos sem pedúnculo variou de 32,8 a 67,9 $\mathrm{N}$ mm, apresentando desvios de 20 a $32 \mathrm{~N} \mathrm{~mm}$.

Observa-se, na Figura 2, que os valores das forças para colapso dos frutos, com e sem pedúnculo, comprimidos a uma velocidade de $0,5 \mathrm{~mm} \mathrm{~min}^{-1}$, são bem próximos; para as outras velocidades, as forças tendem a ser maiores para frutos com pedúnculo e, para cada velocidade de compressão, as energias requeridas para o colapso de frutos com pedúnculo parecem ser maiores que aquelas para o produto sem pedúnculo. As diferenças entre os valores da deformação específica para o colapso de frutos com e sem pedúnculo, parecem ser muito pequenas. Os resultados de testes de comparação de média (Newman Keuls, 5\% de probabilidade), aplicados aos dados experimentais, mostraram que os valores médios das deformações específicas e das energias para colapso dos frutos "cereja" comprimidos com e sem pedúnculo, em cada uma das cinco velocidades, não diferiram significativamente. $\mathrm{O}$ mesmo resultado foi obtido para os valores médios das forças para colapso, exceto quando o produto foi comprimido a velocidade de $10 \mathrm{~mm} \mathrm{~min}^{-1}$, caso em que a força para o colapso de frutos 
com pedúnculo foi maior que aquela requerida para colapso de frutos sem pedúnculo.

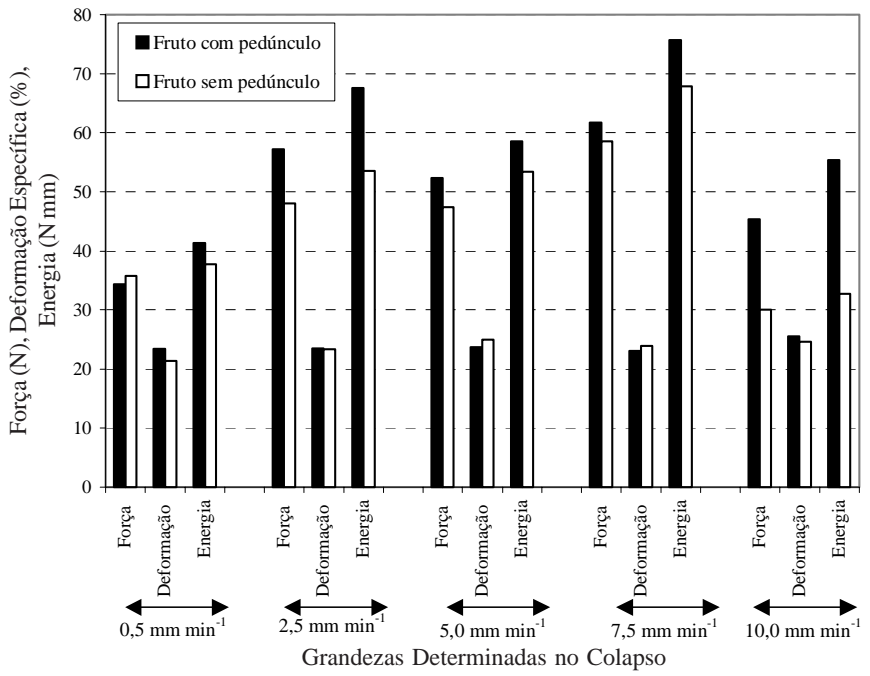

Figura 2. Valores médios de força, deformação específica e energia requeridos para o colapso de frutos de café "cereja", colhidos com e sem pedúnculo, comprimidos a velocidades de $0,5,2,5,5,0,7,5$ e $10,0 \mathrm{~mm} \mathrm{~min}^{-1}$

Inspeção visual dos frutos após cada ensaio de compressão não tornou evidente, muitas vezes, a existência de rachaduras na casca do produto. Quando os frutos sob teste eram "cereja" com pedúnculo, qualquer trinca na casca era fácil de se detectar por causa da liberação de líquido no local. Neste caso, o número de frutos (de um total de 20) comprimidos a velocidades de 0,5 , $2,5,5,0,7,5$ e 10,0 $\mathrm{mm} \mathrm{min}^{-1}$, que apresentaram rachas na casca foi, respectivamente, 9, 9, 10, 6 e 7. O colapso dos outros frutos se deu, provavelmente, na estrutura interna do fruto, enquanto para os frutos "cereja" sem pedúnculo, observou-se que, a medida em que se aumentava a velocidade de compressão, maior quantidade de líquido era eliminada pelo produto na região da casca, danificada pela perda do pedúnculo.

Velocidade de compressão: A Figura 3 apresenta os valores médios das forças, deformações específicas e energias envolvidas no colapso de frutos "cereja", colhidos com e sem pedúnculo, e "verde", em função da velocidade de compressão do produto. As linhas que unem as médias servem apenas para facilitar visualização de tendências. Observa-se que os valores médios das forças e energias tendem a ser maiores para frutos "verdes".

Nota-se, na Figura 3, que não existe tendência definida para o comportamento dos valores das forças, das deformações nem das energias para o colapso do produto com a variação da velocidade de compressão. Modelos polinomiais de primeiro e de segundo graus testados (análise de regressão) não se ajustaram aos dados experimentais.

Quando se focaliza o comportamento do produto comprimido nas diferentes velocidades, constata-se sobreposição dos intervalos de variação de cada uma das grandezas investigadas, podendo ser um indicativo de que a velocidade de compressão não contribuiu de forma significativa para a variação nos valores das grandezas. Valores para as forças, deformações e energias

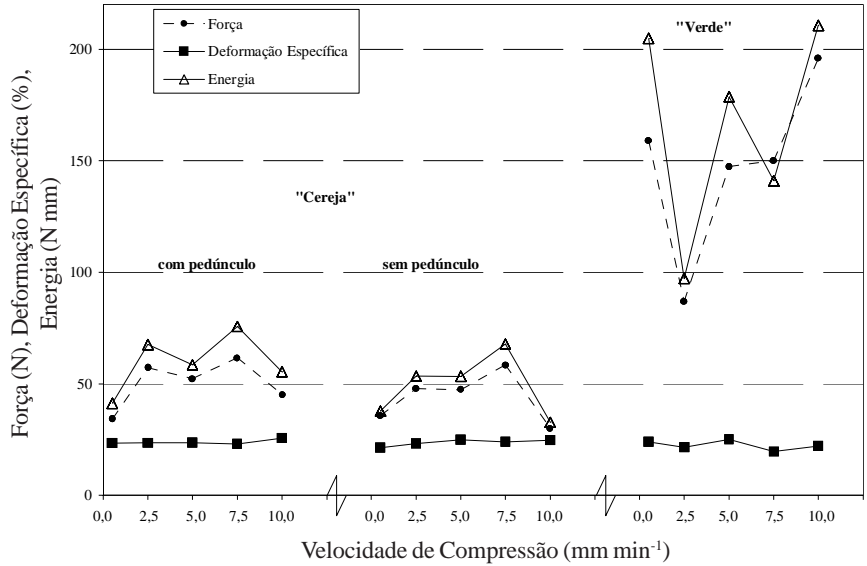

Figura 3. Variação dos valores médios das forças, deformações específicas e energias envolvidas no colapso de frutos "cereja", colhidos com e sem pedúnculo, e "verde", em função da velocidade de compressão do produto

requeridos para o colapso de frutos "verde" e "cereja", com e sem pedúnculo, acompanhados dos respectivos desvios-padrão, quando se considera uma média para todas as velocidades de compressão, são apresentados na Tabela 1. É interessante notar-se que os desvios apresentados pela deformação específica, devidos à variação da velocidade de compressão, são bem menores que os desvios experimentais, e aqueles para força e energia se encontram na faixa dos experimentais.

Tabela 1. Valores médios, com os respectivos desvios-padrão, para forças, deformações e energias requeridos para o colapso de frutos "verde" e "cereja", ao se desconsiderar o efeito da velocidade de compressão

\begin{tabular}{lccc}
\hline \multirow{2}{*}{ Grandeza } & \multicolumn{2}{c}{ "Cereja" } & \multirow{2}{*}{ "Verde" } \\
\cline { 2 - 3 } & $\mathrm{CP}$ & $\mathrm{SP}$ & \\
\hline Força (N) & $50 \pm 11$ & $44 \pm 11$ & $147 \pm 39$ \\
Deformação específica (\%) & $24 \pm 1$ & $24 \pm 1$ & $22 \pm 2$ \\
Energia (N mm) & $59 \pm 13$ & $49 \pm 14$ & $167 \pm 48$ \\
\hline
\end{tabular}

CP - com pêndulo; SP - sem pêndulo

Testes para comparação de médias, aplicados aos dados experimentais, mostraram que: (a) os valores médios das deformações de frutos "verde", comprimido em cada uma das velocidades, não diferiram significativamente daqueles para frutos "cereja"; exceção se faz à compressão do produto a uma velocidade de 7,5 $\mathrm{mm} \mathrm{min}^{-1}$, caso em que a deformação percebida pelos frutos na coloração "verde", foi a menor; e (b) que as forças envolvidas no colapso de frutos "verdes" foram sempre maiores que aquelas requeridas para frutos "cereja", independente da velocidade de compressão do produto. $\mathrm{O}$ mesmo fato ocorreu para a energia requerida para o colapso dos frutos "verde" e "cereja", a não ser para compressões a uma velocidade de $2,5 \mathrm{~mm} \mathrm{~min}^{-1}$, quando não foram detectadas diferenças significativas entre os requerimentos energéticos dos frutos.

Célula de carga e tamanho da amostra: Os valores das forças para o colapso dos frutos, nos vários testes experimentais, variaram de 36,5 a 290,3 N, para frutos "verde", de 14,8 a 201,8 N, para "cereja" com pedúnculo e de 6,9 a 117,8 N, para "cereja" sem pedúnculo. Baseando-se na faixa total de variação dos 
valores desta grandeza, verifica-se que a utilização de uma célula de carga de $490 \mathrm{~N}$ seria o mais adequado para se obter maior exatidão na determinação das forças de colapso experimentais.

Para cada combinação de estádio de maturação - presença ou não de pedúnculo - velocidade de compressão, determinouse o tamanho aproximado da amostra (número de frutos a serem comprimidos) necessário para se obter a força média para o colapso do produto, com $95 \%$ de confiança e exatidão especificada (erros relativos na faixa de 1 a 10\%, em intervalos de $1 \%$ ). Nestas determinações e em cada caso, o desvio-padrão experimental (compressão de 20 frutos) foi usado como estimativa da variância da população de frutos de café.

Devido aos grandes desvios experimentais, o número de frutos determinado para compor uma amostra foi muito grande. Por exemplo, para se obter um erro relativo de $1 \%$, o tamanho da amostra variou desde 2073, para frutos "verdes" comprimidos a $10 \mathrm{~mm} \mathrm{~min}^{-1}$, até 17.693, para frutos “cereja”, com pedúnculo, comprimidos a 7,5 $\mathrm{mm} \mathrm{min}^{-1}$. Observou-se que, apesar dos requerimentos do tamanho das amostras diminuírem, como era esperado, a medida em que se diminuiu a exatidão desejada nos resultados das forças, estes requerimentos variaram com o estádio de maturação dos frutos, com a presença ou não do pedúnculo durante a compressão e com a velocidade de compressão.

A Figura 4 mostra tamanho das amostras e número de frutos a serem comprimidos em cada combinação, para assegurar com 95\% de confiança, que o erro percentual, ao se estimar a força verdadeira pela força média amostral, seja igual ou menor que 5 e $10 \%$. Nota-se que, para um erro de $10 \%$ ao se usar uma velocidade de compressão de $5 \mathrm{~mm} \mathrm{~min}^{-1}$, um tamanho de amostra igual a 50 frutos, independente do estádio de maturação do produto, seria adequado.

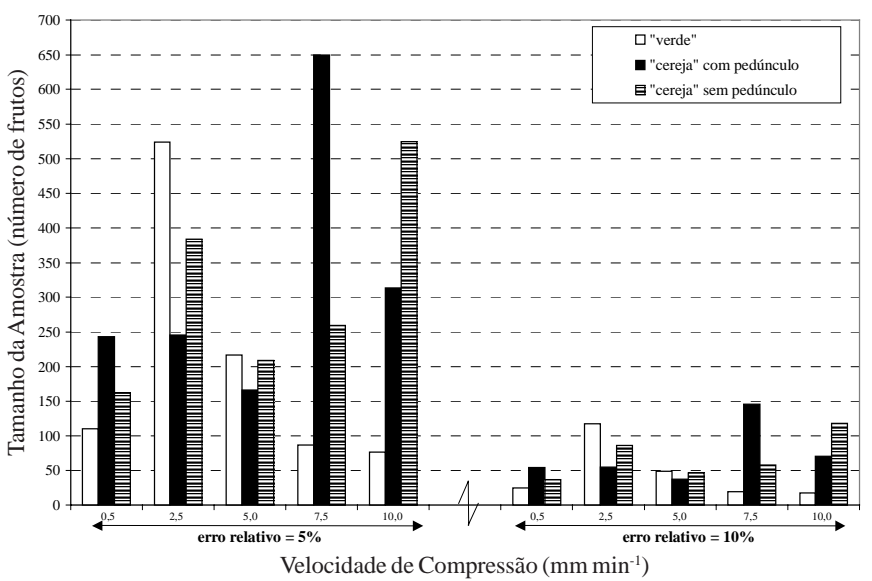

Figura 4. Tamanho da amostra (número de frutos) requerido para, com erros relativos de 5 e $10 \%$, obter-se valores médios amostrais das forças envolvidas no colapso de frutos "verde" e cereja", colhidos com e sem pedúnculo, em função da velocidade de compressão do produto

\section{Experimento 2}

Posição de compressão e grau de maturação do fruto: Com base nos resultados obtidos no experimento anterior, os ensaios de compressão neste experimento, foram realizados com 50 frutos, de cada combinação "grau de maturação-posição de compressão", a uma velocidade de $5 \mathrm{~mm} \mathrm{~min}^{-1}$, cujos frutos se encontravam em três estádios de maturação: cereja, a 69,9 $90,4 \%$ b.u.; verdoengo, a $75,7 \pm 0,9 \%$ b.u., e verde, a $72,0 \pm 0,8 \%$ b.u. e foram comprimidos segundo as três posições esquematizadas na Figura 1. A Figura 5 apresenta os valores médios das dimensões do produto segundo o eixo de compressão, das forças, das deformações específicas e das energias requeridas para o colapso dos frutos. Os valores das dimensões foram multiplicados por vinte, e das deformações específicas por dez, para melhor visualização na escala utilizada, comum a todas as grandezas.

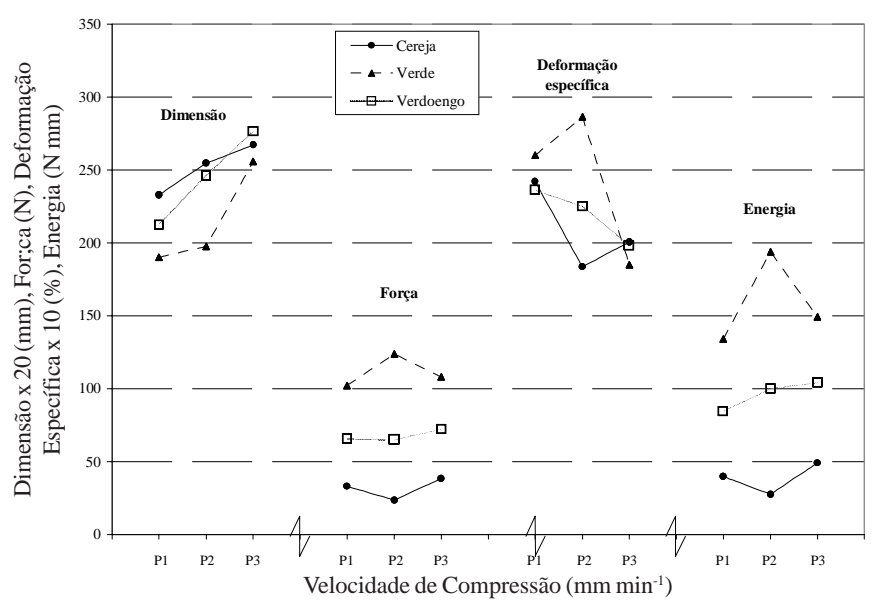

Figura 5. Valores de dimensão (segundo eixo de compressão), força, deformação específica e energia, requeridas para colapso de frutos de café, a variados graus de maturação, comprimidos em diferentes posições

Pode-se observar, na Figura 5, grande variação nos valores das forças e energias requeridas para o colapso dos frutos de café. As forças médias para o colapso do produto variaram de 5,7 $\mathrm{N}$ (frutos "cereja", comprimidos na posição P1) até $231,8 \mathrm{~N}$ (frutos "verde", comprimidos na posição P2) enquanto os valores de energia variaram de 2,3 $\mathrm{N} \mathrm{mm}$ (frutos "verde", comprimidos na posição P3) até $557,9 \mathrm{~N} \mathrm{~mm}$ (frutos "verde", comprimidos na posição P2). Os requerimentos de forças e energias para o colapso do produto parecem depender do grau de maturação dos frutos e existe tendência decrescente desses requerimentos para frutos, na seguinte ordem seqüencial: "verde", "verdoengo" e "cereja". Em média, para todas as posições de compressão, as forças requeridas pelo fruto "verde" foram 1,6 vez maiores que aquelas para frutos "verdoengo" que, por sua vez, são 2,1 vezes maiores que as forças para o colapso do fruto "cereja". Nota-se que, para frutos com um mesmo grau de maturação, existe uma superposição dos intervalos de variação das grandezas investigadas, quando a posição de compressão é variada, o que pode ser forte indicativo da variável não ser significante no processo de colapso.

A deformação específica média para o colapso do produto variou de 5,7\% (frutos verdes, comprimidos na posição P3) até $40,7 \%$ (frutos verdes, comprimidos na posição P2). Na Figura 5 observa-se que parece não haver influência do grau de maturação do produto para compressões nas posições P1 e P2; entretanto, quando o fruto é comprimido na posição P2, parece haver tendência da deformação do fruto "verde" ser maior que a do "verdoengo" que, por sua vez, parece ser maior que a do fruto "cereja". 
Os dados experimentais foram submetidos a testes de comparação de média para investigar a maneira pela qual os valores de força, deformação específica e energia, para o colapso do produto, eram afetados pela posição de compressão do fruto, quando em determinado grau de maturação, e pelo estádio de maturação do produto ao ser comprimido em certa posição. As análises mostraram que:

- as forças e energias requeridas para o colapso de frutos "cereja" e "verdoengo" independem da posição de compressão do produto; entretanto, para frutos "verde", os requerimentos dessas grandezas foram os maiores para a posição P2, não tendo sido detectada diferença significativa para as outras posições;

- os valores da deformação específica para o colapso dos frutos nos três estádios de maturação, foram afetados de maneira diferenciada, pela posição do produto, durante a compressão. Para frutos "verde", os valores máximo e mínimo de deformação aconteceram para as posições P2 e P3, respectivamente. As maiores deformações do fruto "verdoengo" ocorreram quando estes foram comprimidos nas posições P1 e P2; já para os frutos "cereja", os valores da deformação do produto comprimido na posição P1 foram os maiores; não houve diferença significativa para a deformação nas outras posições;

- independente da posição de compressão, as forças e energias requeridas para o colapso de frutos "verde" foram maiores que aquelas para frutos "verdoengo" que, por sua vez, foram superiores àquelas para o produto "cereja";

- o grau de maturação dos frutos não influenciou os valores das deformações específicas quando o produto foi comprimido nas posições P1 e P3, mas para a compressão na posição P2, os valores máximo e mínimo de deformação ocorreram para frutos "verde" e "cereja", respectivamente.

\section{CONCLUSÕES}

1. Os valores das forças, deformações específicas e energias requeridas para o colapso de frutos de café "cereja”, para cada velocidade de compressão, não foram afetados pela presença do pedúnculo no produto; exceção se faz para a velocidade de compressão de $10 \mathrm{~mm} \mathrm{~min}^{-1}$, quando os requerimentos de força foram maiores para o produto com o pedúnculo.

2. A velocidade de compressão parece não afetar as grandezas investigadas.

3. Os valores das deformações específicas para o colapso dos frutos "verde" e "cereja", para cada velocidade de compressão, não diferem, porém os requerimentos de forças e energias para frutos "verde" foram maiores que aqueles para os "cereja".

4. Valores confiáveis de forças para colapso do produto, independentemente do estádio de maturação, são obtidos ao serem usadas uma célula de carga de 490 N (na máquina de ensaios), uma amostra composta de 50 frutos e velocidade de

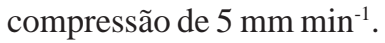

5. Os valores das forças e energias requeridas para o colapso do produto, foram influenciados pela posição de compressão apenas para frutos "verdes".

6. Os valores da deformação específica para o colapso dos frutos nos três estádios de maturação, foram afetados pela posição do produto durante a compressão.

7. As forças e energias requeridas para o colapso de frutos "verde", independente da posição de compressão, foram maiores que aquelas para frutos "verdoengo" que, por sua vez, foram superiores àquelas, para o produto "cereja".

8. O grau de maturação dos frutos não influenciou os valores de suas deformações específicas, quando o produto foi comprimido nas posições P1 e P3;.entretanto, para a compressão na posição P2, as maiores deformações ocorreram para frutos "verde", e as menores para o produto "cereja".

\section{LITERATURA CITADA}

Agricultural Engineers Yearbook of Standards. ASAE, St. Joseph, MI, 1995.

Amorim, H.V.; Cruz, A.R.; Gutierrez, L.E.; Teixeira, A.A.; Mello, M.; Oliveira, G.D. Transformações químicas e estruturais durante a deterioração da qualidade do café. In: Congresso Brasileiro de Pesquisas Cafeeiras. Resumos... Guarapari, ES. IBC/GERCA, 1977, p.15-18.

Gupta, R.K.; Das, S.K. Fracture resistance of sunflower seed and kernel to compressive loading. Journal of Food Engineering, Dublin, v.46, n.1, p.1-8, 2000.

Henry, Z.A.; Su, B.; Zhang, H. Resistance of soya beans to compression. Journal of Agricultural Engineering Research, London, v.76, n.2, p.175-181, 2000.

Li, Y.; Zhang, Q.; Puri, V.M.; Manbeck, H.B. Physical properties effect on stress-strain behavior of wheat en masse - Part I. Load response dependence on initial bulk density and moisture content. Transactions of the ASAE, St. Joseph, v.32, n.1, p.194-202, 1989.

Mohsenin, N.N. Physical properties of plant and animal materials. 2.ed. New York: Gordon and Breach Science Publishers, 1978. 742p.

Prussia, S.E.; Campbell, D.T. Apparent modulus elasticity of maturing pecans. Transactions of the ASAE, St. Joseph, v.28, n.4, p.1290-1296, 1985.

Silva, J.S.; Berbert, P.A. Colheita, secagem e armazenagem de café. Viçosa: Aprenda Fácil Editora, 1999. 146p.

Zhang, Q.; Li, Y; Puri, V.M.; Manbeck, H.B. Physical properties effect on stress-strain behavior of wheat en masse - Part II. Constitutive elastoplastic parameter dependence on initial bulk density and moisture content. Transactions of the ASAE St. Joseph, v.32, n.1, p.203-209, 1989. 